$13^{\text {th }}$ International Conference on AEROSPACE SCIENCES \& AVIATION TECHNOLOGY, ASAT-13, May 26 - 28, 2009, E-Mail: asat@mtc.edu.eg Military Technical College, Kobry Elkobbah, Cairo, Egypt Tel : +(202) $24025292-24036138$, Fax: +(202) 22621908

\title{
Design of the Rotor Blade for a Wind Mill
}

\section{Daniel Svrcek $^{*}$}

Abstract: The design of a rotor blade requires comprehensive information of the particular wind-mill location. The design itself consists in determining the aerodynamic and geometric characteristics of the rotor blade (local chords, blade twist, airfoils). The blade design has to satisfy the design conditions, gain high aerodynamic efficiency and simultaneously take into consideration blade structural constraints (diameter, number of blades, revolutions).

The paper deals with the design of aerodynamic characteristics of the rotor blade of a wind mill in a chosen design point. The design is specified for the rotor of the diameter 20.4 [m] and revolutions $0.728\left[\mathrm{~s}^{-1}\right]$.

Keywords: Wind mill, Propellers, Aerodynamic design

\begin{tabular}{|c|c|c|}
\hline \multicolumn{3}{|c|}{ Nomenclature } \\
\hline$C$ & {$[-]$} & Correction factor (effect of number of blades) \\
\hline$D$ & {$[m]$} & Propeller diameter \\
\hline$Q$ & {$[N]$} & Drag \\
\hline$L$ & {$[N]$} & Lift \\
\hline$N$ & {$[N]$} & Circumferential force (plane of rotation) \\
\hline$P$ & {$[W$} & Power \\
\hline$R$ & {$[m]$} & Propeller radius \\
\hline$S$ & {$\left[m^{2}\right]$} & Propeller disc area \\
\hline$T$ & {$[N]$} & Thrust \\
\hline$W$ & {$[W]$} & Power developed by the rotor \\
\hline$\beta_{0}$ & {$[0]$} & Flow angle \\
\hline$\beta_{1}$ & {$[0]$} & Flow angle (induced velocity included) $w_{1}$ \\
\hline$\Gamma$ & {$\left[m^{2} \cdot s^{-1}\right]$} & Circulation \\
\hline$b$ & {$[m]$} & Local blade chord \\
\hline$C_{L}$ & {$[-]$} & Lift coefficient \\
\hline$C_{P}$ & {$[-]$} & Power coefficient \\
\hline
\end{tabular}

* Senior lecturer, Ing. Daniel Švrček, PhD., Slovak University of Technology in Bratislava, Faculty of Materials Science and Technology in Trnava, Paulinska 16, 91724 Trnava, Slovak Republic, Tel.: +42133 5511601, e-mail: agrolet@stonline.sk 


\begin{tabular}{|c|c|c|}
\hline$c_{Q}$ & {$[-]$} & Drag coefficient \\
\hline$C_{T}$ & {$[-]$} & Thrust coefficient \\
\hline$c_{L}^{\alpha}$ & {$[-]$} & Lift curve slope \\
\hline$h_{1}, h_{2}$ & $k[-]$ & Iteration constants \\
\hline$n$ & {$\left[s^{-1}\right.$} & Revolutions \\
\hline$r$ & {$[m]$} & Local blade radius \\
\hline$t$ & {$[m]$} & Airfoil thickness \\
\hline$u_{0}$ & {$\left[m \cdot s^{-1}\right]$} & Circumferential velocity \\
\hline$u_{1}$ & $\left.m \cdot s^{-1}\right]$ & Circumferential velocity (incl. induced quantities) $u_{i}$ \\
\hline$v_{0}$ & {$\left[m \cdot s^{-1}\right]$} & Velocity in front of the propeller (flight speed) \\
\hline$v_{1}$ & {$\left[m \cdot s^{-1}\right]$} & Axial velocity component (incl. induced velocity) $v_{i}$ \\
\hline$v_{i}$ a $u_{i}$ & {$\left[m \cdot s^{-1}\right]$} & Induced velocity \\
\hline$w_{0}$ & {$\left[m \cdot s^{-1}\right]$} & Resulting velocity \\
\hline$w_{1}$ & {$\left[m \cdot s^{-1}\right]$} & Resulting velocity (induced velocity included) \\
\hline$z$ & {$[-]$} & Number of blades \\
\hline$\alpha$ & {$[0$} & Angle of attack \\
\hline$\alpha_{0}$ & {$[0$} & Zero lift angle \\
\hline$\eta$ & {$[-, \%]$} & Efficiency \\
\hline$\varphi$ & {$[0]$} & Angle of blade twist (setting angle) \\
\hline$\lambda$ & {$[-]$} & Advance ratio \\
\hline$\mu$ & {$[-]$} & Airfoil drag/lift ratio \\
\hline$\omega$ & {$\left[S^{-1}\right]$} & Angular velocity \\
\hline$\rho$ & {$\left[\mathrm{kg} \cdot \mathrm{m}^{-3}\right]$} & Mass density of air \\
\hline
\end{tabular}

\section{Introduction}

The paper describes the design of a rotor blade of a wind-mill which gives high aerodynamic efficiency at the design point. Velocity components and local force components are expressed as functions of circulation the distribution of which giving maximum efficiency is calculated. The link between geometric/aerodynamic characteristics makes possible to determine structural parameters of the rotor blade.

\section{Design Specification.}

The selection of the design point for the rotor requires comprehensive information of the conditions at the particular location of the wind-mill. The design parameters include wind velocity (average) and the structural parameters - the diameter, number of blades, and rotor revolutions.

In the design process it is to determine the rotor geometric and aerodynamic characteristics (distribution of blade chords, distribution of blade twist, airfoils). These parameters have to conform to the design specifications and moreover achieve the aerodynamic efficiency as high as possible.

The essential propeller characteristics are introduced as follows: 
Advance ratio

$$
\lambda=\frac{v_{0}}{n \cdot D}
$$

Thrust and drag

$$
\begin{aligned}
& T=\rho \cdot n^{2} \cdot D^{4} \cdot c_{T} \\
& Q=\rho \cdot n^{2} \cdot D^{4} \cdot c_{Q}
\end{aligned}
$$

Power

$$
P=\rho \cdot n^{3} \cdot D^{5} \cdot c_{P}
$$

The rotor efficiency is determined by the thrust and power coefficients and the advance ratio[6]

$\eta=\lambda \cdot \frac{c_{T}}{C_{P}} \rightarrow \max$

\section{Set of Propeller Equations}

In the following relations the indices 0,1 describe the flow in front of a propeller (index , ,6 ) and in the propeller disc (index ," “), as indicated in Fig.1.

The relation between the local lift values and the total lift can be expressed as

$$
\begin{aligned}
& L=\frac{1}{2} \rho \cdot w_{1}^{2} \cdot c_{L} \cdot S=\frac{1}{2} \rho \cdot \int_{0}^{R} w_{1(r)}^{2} \cdot c_{L(r)} \cdot b_{(r)} \cdot d r \\
& d L=\frac{1}{2} \rho \cdot w_{1}^{2} \cdot c_{L} \cdot b \cdot d r
\end{aligned}
$$

Introducing the concept of circulation and the Joukovski - Kutta theorem makes possible to write relations

$$
\begin{aligned}
& \Gamma=\frac{1}{2} \rho \cdot w_{1} \cdot c_{L} \cdot b \\
& d L=\rho \cdot w_{1} \cdot \Gamma \cdot d r
\end{aligned}
$$

These terms when inserted into (6) give the lift resultant which depends on the distribution of circulation along a propeller blade.

The previous procedure transfers the task of gaining maximum efficiency to the problem of determining the optimum distribution of circulation. 
To facilitate the numerical procedures the non-dimensional quantities are introduced

$$
\bar{\Gamma}=\frac{\Gamma}{4 . \pi \cdot R^{2} \cdot \omega} \quad \bar{b}=\frac{z \cdot b}{4 \cdot \pi \cdot R} \quad \bar{r}=\frac{r}{R} \quad \bar{v}_{1}=\frac{v_{1}}{R \cdot \omega} \quad \bar{u}_{1}=\frac{u_{1}}{R \cdot \omega}
$$

where the velocity components are indicated in Fig.1. [5]

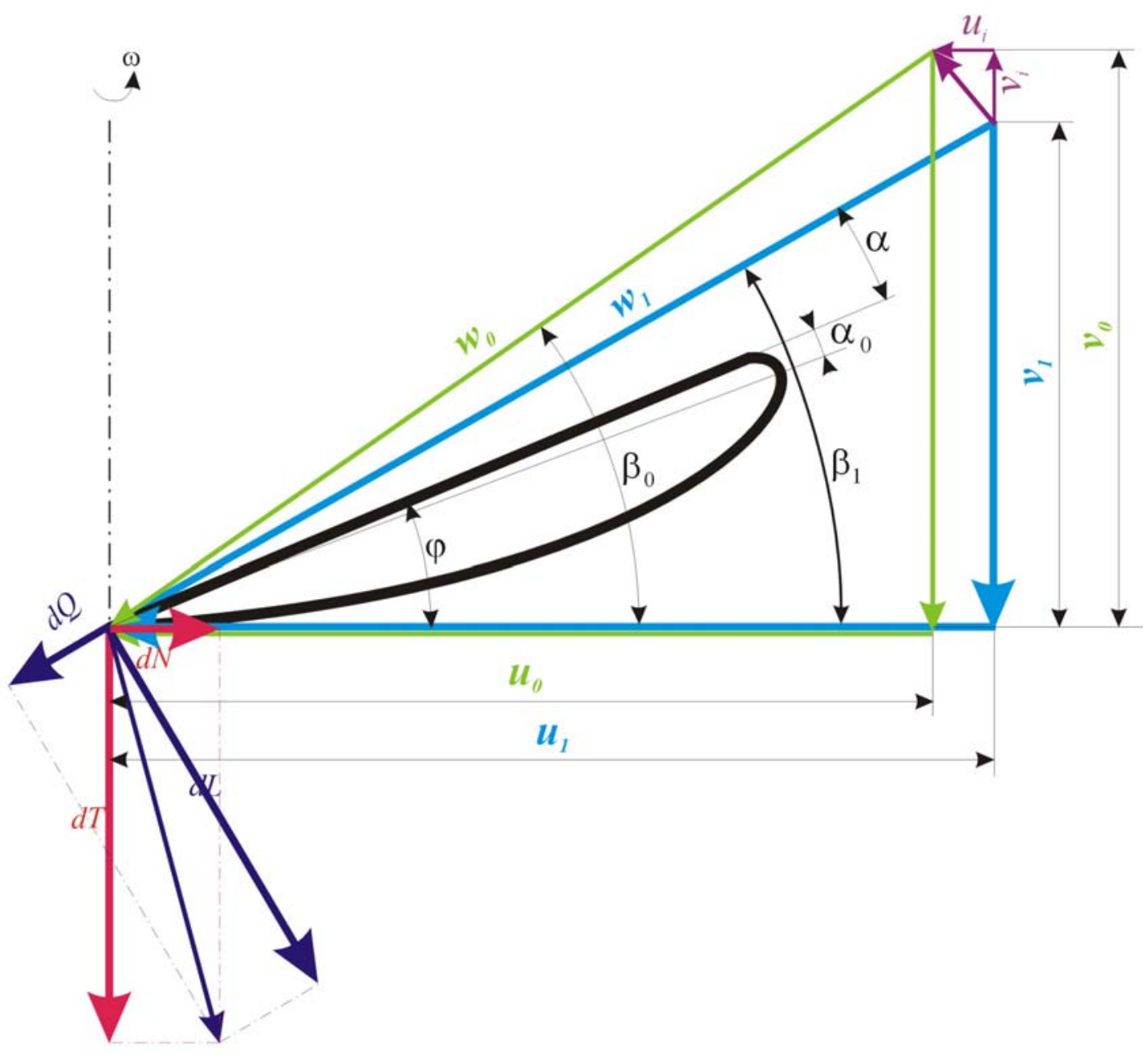

Fig. 1 Velocity and force components

\section{Velocity Relations at a Local Blade Section}

The resulting velocity at the local radius including induced effects is composed of axial and tangential components

$$
\begin{aligned}
& \bar{w}_{1}=\sqrt{\left(\bar{u}_{1}^{2}+\bar{v}_{1}^{2}\right)} \\
& \bar{u}_{1}=\bar{u}_{0}+\bar{u}_{i} \\
& \bar{v}_{1}=\bar{v}_{0}+\bar{v}_{i}
\end{aligned}
$$


or by means of (12) and (13)

$$
\bar{u}_{1}=\bar{r}+\bar{u}_{i}
$$

$$
\bar{v}_{1}=\frac{\lambda}{. \pi}+\bar{v}_{i}
$$

The simplest vortex system of a propeller is represented by a system of concentric vortex cylinders with a continuous distribution of circulation (by Joukovski) which gives the following relations for velocity components as functions of circulation

$$
\begin{aligned}
& \bar{u}_{1}=\bar{r}+\frac{\bar{\Gamma}}{\bar{r}} \\
& \bar{v}_{1}=\frac{\lambda}{2 . \pi}+\sqrt{\left(\left(\frac{\lambda}{2 . \pi}\right)^{2}-\bar{\Gamma} \cdot(1+\bar{\Gamma})\right)}
\end{aligned}
$$

The vortex system of question does not fully conform to experimental results because it strictly corresponds to the infinite number of blades. As a consequence the error increases with the advance ratio, the propeller power loading and the decrease in number of blades.

It is recommended to improve the error by a correction factor depending on the advance ratio and number of blades. [2]

$$
C=1+1,803 \cdot\left(\frac{1}{z}\right)^{1,16} \cdot \lambda^{2}-0,459 \cdot\left(\frac{1}{z}\right)^{1,062} \cdot \lambda^{3}+0,0243 \cdot\left(\frac{1}{Z}\right)^{0,835} \cdot \lambda^{4}
$$

Substituting into (16), (17) the velocity components become

$$
\begin{aligned}
& \bar{u}_{1}=\bar{r}+C \cdot \frac{\bar{\Gamma}}{\bar{r}} \\
& \bar{v}_{1}=\frac{\lambda}{2 \cdot \pi} \cdot(2-C)+C \cdot \sqrt{\left(\left(\frac{\lambda}{2 \cdot \pi}\right)^{2}-\bar{\Gamma} \cdot(1+\bar{\Gamma})\right)}
\end{aligned}
$$

The following step is to specify links between kinematic and structural quantities by the geometric parameters (angle of blade twist, local sectional chord, airfoil thickness ) to conform the flow relations (Fig. 1)

$$
\begin{aligned}
& \beta_{1}=\operatorname{arctg} \frac{\bar{v}_{1}}{\bar{u}_{1}} \\
& \varphi=\beta_{1}-\alpha \\
& c_{L}=c_{L}^{\alpha}\left[\operatorname{arctg} \frac{\bar{v}_{1}}{\bar{u}_{1}}-\left(\varphi+\alpha_{0}\right)\right]
\end{aligned}
$$


By analyzing the previous relations it is possible to get to the conclusion that the structural parameters $\varphi, b$ a $t$ of the propeller at the particular blade section are determined by the local value of circulation $\bar{\Gamma}$. [3]

\section{Distribution of Optimum Circulation}

Based on the previous considerations on velocity and geometric relations the task to calculate and design the propeller blade is simplified to the determination of optimum distribution of circulation.

The specific method to solve this problem and utilize its result for a real propeller design follows the procedures described in Ref. [3]

An iteration method was used where the successive steps lead to the final value of local circulation. The numerical process is terminated when the accuracy criterion for two consecutive steps is satisfied $\left|\bar{\Gamma}-\bar{\Gamma}_{(-1)}\right| \leq 1.10^{-4}$.

Iteration constants

$$
h_{1}=\frac{\lambda}{2 . \pi} \cdot(2-C)+C \cdot \frac{\left(\frac{\lambda}{2 \cdot \pi}\right)^{2}+\frac{3 \cdot \bar{\Gamma}_{(-1)}}{2}}{\sqrt{\left[\left(\frac{\lambda}{2 . \pi}\right)^{2}+\bar{\Gamma}_{(-1)}\right]}} \quad h_{2}=C \cdot \frac{\left(\frac{\lambda}{2 \cdot \pi}\right)^{2}+\frac{3 \cdot \bar{\Gamma}_{(-1)}}{4}}{\sqrt{\left[\left(\frac{\lambda}{2 . \pi}\right)^{2}+\bar{\Gamma}_{(-1)}\right]}} \quad \sigma=\frac{k \cdot \bar{r}-\mu}{1+k \cdot \mu \cdot \bar{r}}
$$

form auxiliary quantities for the final relation

$$
\bar{\Gamma}=\bar{r} \cdot\left(\frac{\bar{r}+\sigma \cdot\left(h_{1}-h_{2} \cdot \bar{\Gamma}_{(-1)}\right)}{2 \cdot C-\bar{r} \cdot \sigma \cdot h_{2}}\right)
$$

The calculated values represent the optimum distribution of circulation directly giving the distribution of local chords and setting angles. Moreover it enables to calculate even the thrust and power coefficients. [2]

\section{Design of the Rotor Blade}

The method described above was applied to the blade design conforming to the demand of the VEZ Kosice comp.( East Slovakia Energy Ent.). The blade design and its realization were intended as a replacement for an older type of a wind- mill.

The given input data

$$
D=20,40[\mathrm{~m}], \quad v_{0}=8,00\left[\mathrm{~m} \cdot \mathrm{s}^{-1}\right], \quad n=0,728\left[\mathrm{~s}^{-1}\right]=43,70\left[\mathrm{~min}^{-1}\right]
$$

determine $\lambda=0,5384$ and $\mathrm{C}=1.088$.

Subsequently the optimum distribution of circulation was calculated giving the process in Fig. 2. 


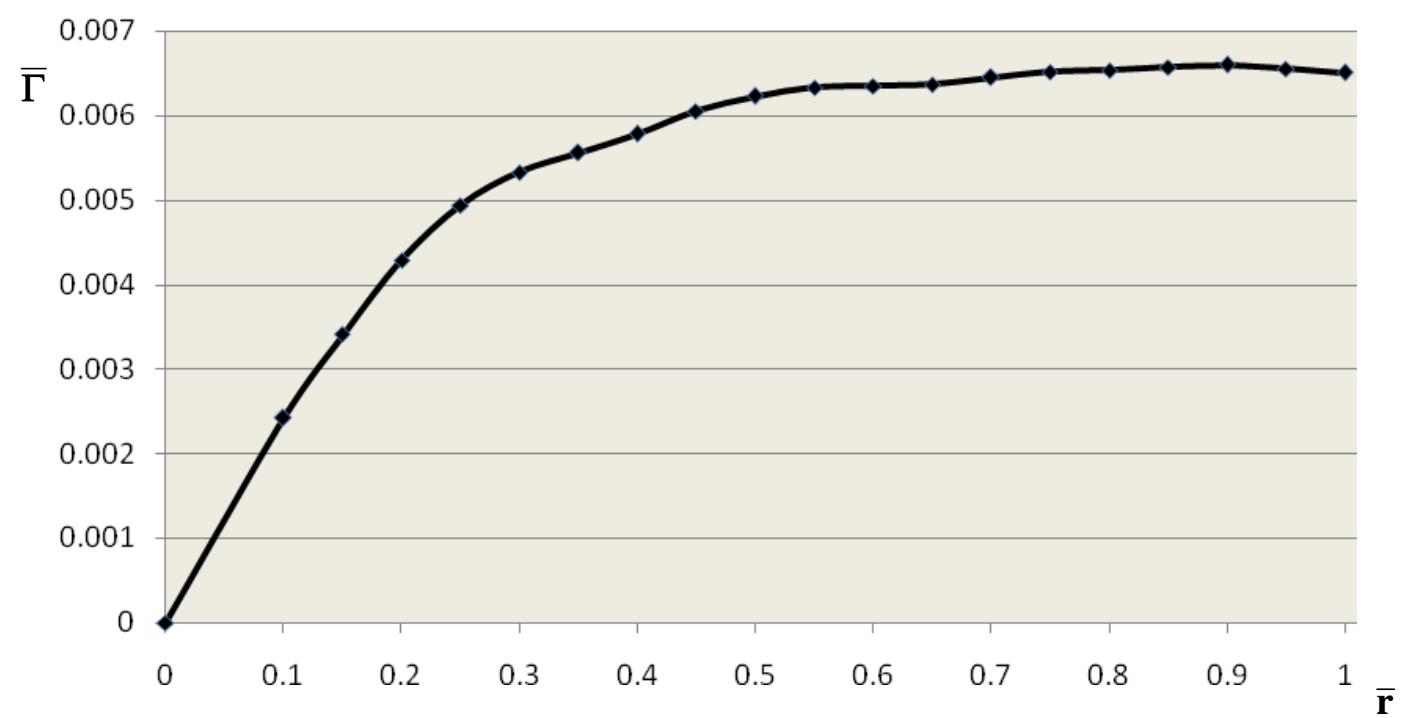

Fig.2. Distribution of circulation $\bar{\Gamma}$ along the propeller blade

Table 1. contains the values of circulation and velocity components (19), (20), (11). The next step consists in calculating the lift distribution $c_{L} \cdot \bar{b}$ from (8) which in connection with the characteristics of the RAF 6 airfoil leads to resulting geometric parameters of the designed blade (Table 2.), Figs. 3 \& 4 ..

Table 1. Distribution of circulation and velocity components on the propeller blade

\begin{tabular}{|l|l|l|l|l|}
\hline \multicolumn{1}{|c|}{$\bar{r}$} & \multicolumn{1}{c|}{$\bar{\Gamma}$} & \multicolumn{1}{c|}{$\bar{u}_{1}$} & \multicolumn{1}{c|}{$\bar{v}_{1}$} & $\bar{w}_{1}$ \\
\hline 0 & 0 & 0 & 0 & 0 \\
\hline 0.1 & 0.00242 & 0,12420 & 0,155816 & 0,19926 \\
\hline 0.15 & 0.00341 & 0,17273 & 0,148313 & 0,22767 \\
\hline 0.2 & 0.00429 & 0,22148 & 0,140725 & 0,26240 \\
\hline 0.25 & 0.00494 & 0,26977 & 0,134445 & 0,30141 \\
\hline 0.3 & 0.00533 & 0,31777 & 0,130210 & 0,34342 \\
\hline 0.35 & 0.00556 & 0,36578 & 0,127982 & 0,38752 \\
\hline 0.4 & 0.00578 & 0,41429 & 0,125606 & 0,43291 \\
\hline 0.45 & 0.00605 & 0,46329 & 0,122111 & 0,47911 \\
\hline 0.5 & 0.00623 & 0,51247 & 0,118330 & 0,52596 \\
\hline 0.55 & 0.00633 & 0,56152 & 0,116763 & 0,57353 \\
\hline 0.6 & 0.00635 & 0,61059 & 0,116411 & 0,62159 \\
\hline 0.65 & 0.00637 & 0,65981 & 0,116116 & 0,66995 \\
\hline 0.7 & 0.00645 & 0,70921 & 0,114880 & 0,71845 \\
\hline 0.75 & 0.00652 & 0,75869 & 0,113592 & 0,76715 \\
\hline 0.8 & 0.00654 & 0,80818 & 0,113156 & 0,81606 \\
\hline 0.85 & 0.00657 & 0,85773 & 0,112650 & 0,86510 \\
\hline 0.9 & 0.00660 & 0,90734 & 0,112015 & 0,91423 \\
\hline 0.95 & 0.00655 & 0,95690 & 0,112929 & 0,96354 \\
\hline 1 & 0.00651 & 1,00652 & 0,113705 & 1,01292 \\
\hline
\end{tabular}


Table 2. Final aerodynamic and geometric characteristics

\begin{tabular}{|l|l|l|l|r|l|r|}
\hline \multicolumn{1}{|c|}{$\bar{r}$} & \multicolumn{1}{|c|}{$c_{L} . \bar{b}$} & \multicolumn{1}{c|}{$c_{L}$} & $b[m]$ & $t[\%]$ & $\left.\alpha{ }^{o}\right]$ & $\left.\varphi^{o}\right]$ \\
\hline 0 & 0.0 & 0.0000 & & & & \\
\hline 0.1 & 0.02429 & 0.6278 & 1.240 & 23.00 & & \\
\hline 0.15 & 0.02996 & 0.8020 & 1.197 & 22.16 & & \\
\hline 0.2 & 0.03274 & 0.9085 & 1.155 & 21.33 & 4.53 & 27.89 \\
\hline 0.25 & 0.03279 & 0.9441 & 1.113 & 20.50 & 4.98 & 21.50 \\
\hline 0.3 & 0.03105 & 0.9301 & 1.070 & 19.66 & 4.56 & 17.72 \\
\hline 0.35 & 0.02851 & 0.8887 & 1.028 & 18.83 & 3.63 & 15.65 \\
\hline 0.4 & 0.02641 & 0.8584 & 0.986 & 18.00 & 3.04 & 13.82 \\
\hline 0.45 & 0.02496 & 0.8484 & 0.943 & 17.16 & 2.82 & 11.93 \\
\hline 0.5 & 0.02372 & 0.8438 & 0.901 & 16.33 & 2.66 & 10.33 \\
\hline 0.55 & 0.02210 & 0.8244 & 0.859 & 15.50 & 2.44 & 9.29 \\
\hline 0.6 & 0.02046 & 0.8035 & 0.816 & 14.66 & 2.27 & 8.51 \\
\hline 0.65 & 0.01903 & 0.7881 & 0.774 & 13.83 & 2.24 & 7.74 \\
\hline 0.7 & 0.01795 & 0.7860 & 0.732 & 13.00 & 2.55 & 6.64 \\
\hline 0.75 & 0.01700 & 0.7908 & 0.689 & 12.16 & 2.93 & 5.57 \\
\hline 0.8 & 0.01604 & 0.7946 & 0.647 & 11.33 & 3.23 & 4.73 \\
\hline 0.85 & 0.01519 & 0.8049 & 0.605 & 10.50 & 3.59 & 3.89 \\
\hline 0.9 & 0.01445 & 0.8241 & 0.562 & 9.66 & 4.11 & 2.92 \\
\hline 0.95 & 0.01361 & 0.8389 & 0.520 & 8.83 & 4.68 & 2.04 \\
\hline 1 & 0.01286 & 0.8625 & 0.478 & 8.00 & 5.32 & 1.11 \\
\hline
\end{tabular}

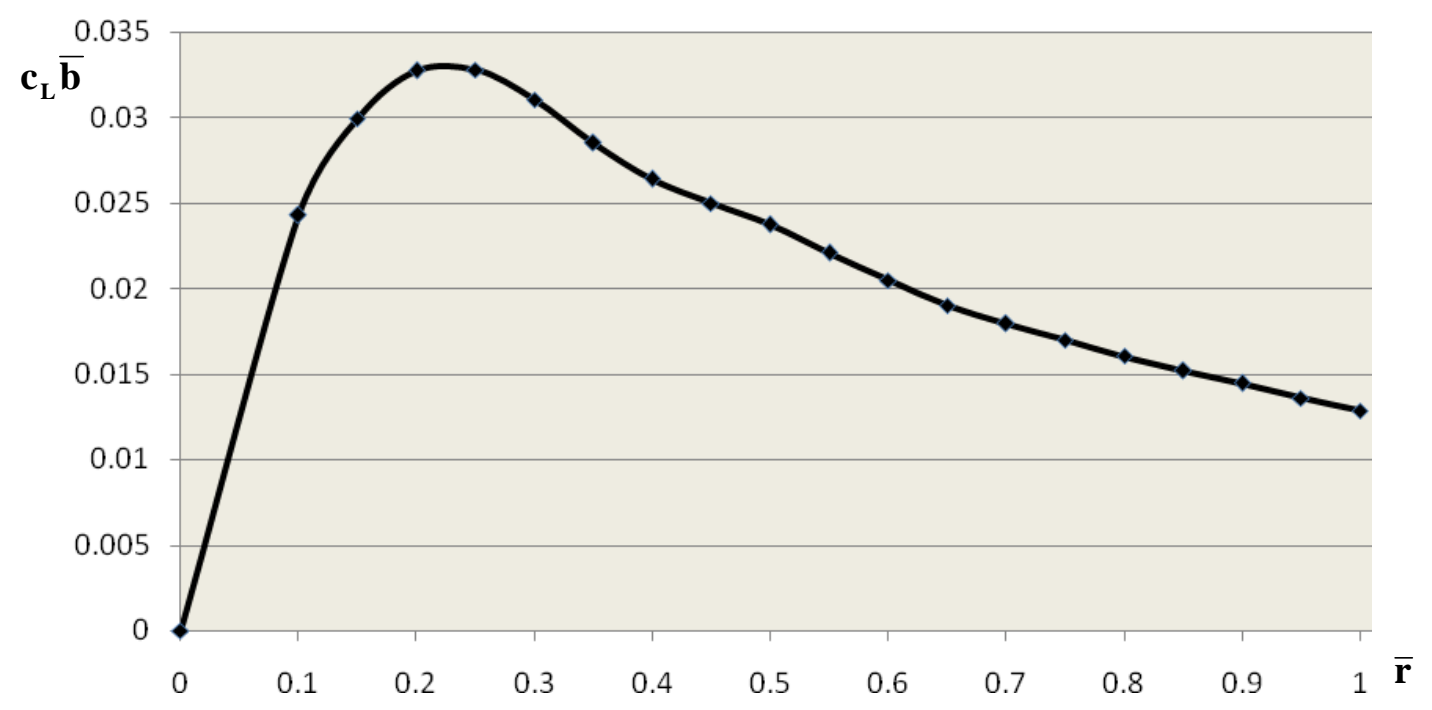

Fig. 3. Lift distribution $c_{L} \cdot \bar{b}$

For the design parameters given as wind velocity $v_{0}=8.00\left[\mathrm{~m} \cdot \mathrm{s}^{-1}\right]$ and rotor revolutions $n=0.73\left[\mathrm{~s}^{-1}\right]$ resulting parameters were calculated 


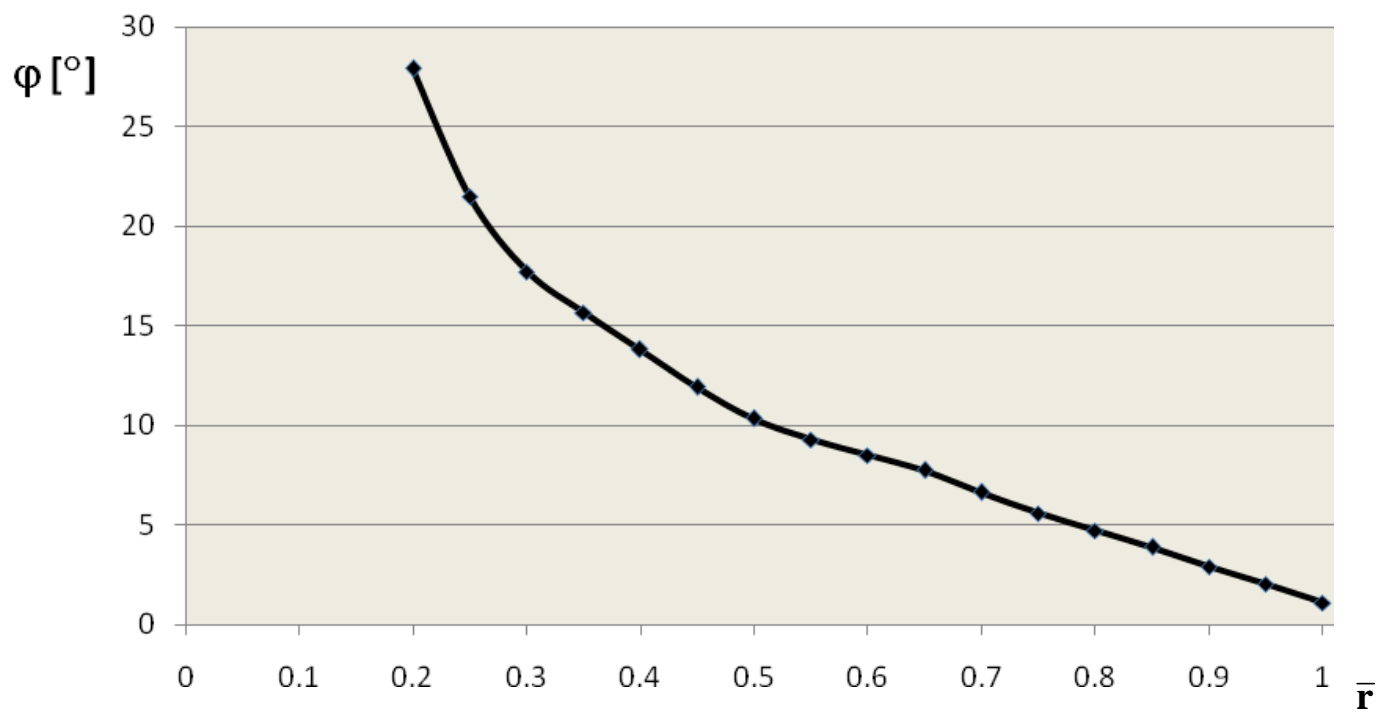

Fig.4. Distribution of blade twist $\varphi\left[{ }^{\circ}\right]$

$C_{T}=0,025569 \eta=44,919[\%] T=11563,3[N] Q=1882,58[N]$

Then the total wind mill power $W$ is calculated from

$W=Z \cdot n \cdot \int_{0}^{R} N_{(r)} \cdot d r$

Calculating the integral (25) by means of values in Table 3 gives the total power $W=47,484[k W]$

\section{Conclusion}

The given method to design the rotor blade results from the distribution of optimum circulation which conforms to the selected design conditions and achieves high aerodynamic efficiency. Circulation relates to the lift distribution which includes geometric and aerodynamic characteristics and makes possible to determine structural parameters of the blade. As an example a particular rotor design is presented giving the rotor geometry, its aerodynamic characteristics and power developed by the wind mill.

\section{Acknowledgement}

The research has been supported by VEGA MS of the Slovak Republic within the project No. $1 / 0837 / 08$ and 1/0256/09. 


\section{References}

[1] FILAKOVSKÝ, K.: Design of a Wind Motor Blade. Transfer VZLÚ, Vol. 4, 2007, pp.1622 (in Czech)

[2] BROŽ, V.: Distribution of Circulation on a Propeller Blade. Zpravodaj VZLÚ, Vol. 5, 1963, pp. 17-23 (in Czech)

[3] BROŽ, V.: Aerodynamic Design of a High Efficiency Propeller. Zpravodaj VZLÚ, Vol. 5, 1966, pp. 324-328 (in Czech)

[4] BROŽ, V., SLAVÍK, S.: Diagrams of Optimum Distributions of Circulation on a Propeller Blade. Zpráva VZLÚ No. V-1348/79, Praha, 1978 (in Czech)

[5] SLAVÍK, S.,: Distribution of Circulation on a Propeller Blade including the Nonlinear Effect of the Number of Blades. Zpravodaj VZLÚ, Vol. 5, 1985, pp. 371-375 (in Czech)

[6] ŠVRČEK, D.: Propellers. Conference on Manufacturing of Microlights Zborník, Bratislava, 1998, pp. 1-10. (in Slovak)

Table 3. Rotor force components and power

\begin{tabular}{|c|c|c|c|c|c|c|}
\hline $\begin{array}{l}R \\
{[m]}\end{array}$ & $\begin{array}{l}L / Z \\
{[N]}\end{array}$ & $\begin{array}{l}Q / z \\
{[N]}\end{array}$ & $\begin{array}{l}T / z \\
{[N]}\end{array}$ & $\begin{array}{l}N / Z \\
{[N]}\end{array}$ & $\begin{array}{l}W / z \\
{[W]}\end{array}$ & $\begin{array}{l}W \\
{[W]}\end{array}$ \\
\hline 10.2 & 0 & 0.0 & 0.0 & 0 & 534.2 & 2136 \\
\hline 9.69 & 286.6 & 7.0 & 286.3 & 13.5 & 1233.7 & 4934 \\
\hline 9.18 & 559.3 & 13.5 & 558.6 & 30.8 & 2041.5 & 8166 \\
\hline 8.67 & 817.7 & 19.6 & 816.2 & 51.4 & 2923.7 & 11694 \\
\hline 8.16 & 1061.5 & 25.4 & 1059.0 & 74.4 & 3828.0 & 15312 \\
\hline 7.65 & 1291.1 & 30.8 & 1287.3 & 99.1 & 4732.7 & 18930 \\
\hline 7.14 & 1505.7 & 36.0 & 1500.3 & 125.6 & 5641.5 & 22566 \\
\hline 6.63 & 1704.8 & 40.9 & 1697.4 & 153.7 & 6529.2 & 26116 \\
\hline 6.12 & 1888.5 & 45.6 & 1878.9 & 182.8 & 7364.7 & 29458 \\
\hline 5.61 & 2057.3 & 50.1 & 2045.2 & 212.2 & 8136.3 & 32545 \\
\hline 5.1 & 2211.3 & 54.5 & 2196.4 & 241.4 & 8833.8 & 35335 \\
\hline 4.59 & 2350.4 & 58.6 & 2332.6 & 270.4 & 9456.6 & 37826 \\
\hline 4.08 & 2474.6 & 62.6 & 2453.6 & 298.8 & 10002.7 & 40010 \\
\hline 3.57 & 2584.1 & 66.6 & 2559.5 & 326.6 & 10471.2 & 41884 \\
\hline 3.06 & 2679.4 & 70.6 & 2651.0 & 353.4 & 10864.8 & 43459 \\
\hline 2.55 & 2761.0 & 74.9 & 2728.4 & 379.7 & 11197.3 & 44789 \\
\hline 2.04 & 2829.4 & 79.5 & 2791.7 & 405.9 & 11477.1 & 45908 \\
\hline 1.53 & 2885.2 & 83.9 & 2841.0 & 432.2 & 11702.1 & 46808 \\
\hline 1.02 & 2929.0 & 87.9 & 2876.4 & 458.0 & 11871.1 & 47484 \\
\hline 0.51 & 2948.0 & 89.7 & 2890.8 & 470.6 & 11871.1 & 47484 \\
\hline
\end{tabular}

